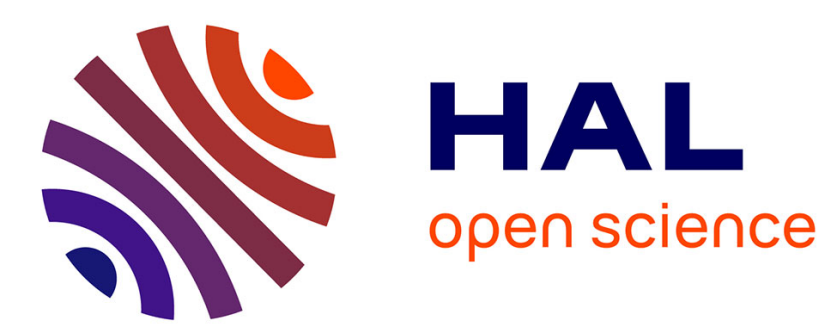

\title{
An Improved Genetic-Based Link Clustering for Overlapping Community Detection
}

\author{
Yong Zhou, Guibin Sun
}

\section{To cite this version:}

Yong Zhou, Guibin Sun. An Improved Genetic-Based Link Clustering for Overlapping Community Detection. 9th International Conference on Intelligent Information Processing (IIP), Nov 2016, Melbourne, VIC, Australia. pp.142-151, 10.1007/978-3-319-48390-0_15 . hal-01614993

\section{HAL Id: hal-01614993 \\ https://hal.inria.fr/hal-01614993}

Submitted on 11 Oct 2017

HAL is a multi-disciplinary open access archive for the deposit and dissemination of scientific research documents, whether they are published or not. The documents may come from teaching and research institutions in France or abroad, or from public or private research centers.
L'archive ouverte pluridisciplinaire HAL, est destinée au dépôt et à la diffusion de documents scientifiques de niveau recherche, publiés ou non, émanant des établissements d'enseignement et de recherche français ou étrangers, des laboratoires publics ou privés. 


\title{
An Improved Genetic-based Link Clustering for Overlapping Community Detection
}

\author{
Yong Zhou ${ }^{1}$, Guibin Sun ${ }^{1}$ \\ 1 School of Computer Science and Technology, China University \\ of Mining and Technology, Xuzhou 221008,China \\ yzhoudcumt. edu.cn \\ sunguibinbesteqq. com
}

\begin{abstract}
The problem of community detection in complex networks has been intensively investigated in recent years. And it was found that the communities of complex networks often overlap with each other. So in this paper, we propose an improved genetic-based link clustering for overlapping community detection. The first, the algorithm changes the node graph into the link graph. The second, the algorithm adopts the genetic algorithm to detect the link communities. The Third, the algorithm transforms the link communities into the node communities. Automatically, the nodes, which are linked with edges belonged to different link communities, will be the overlapping nodes. The last, in order to improve the quality of community detection, we define an effective method to solve the "excessive overlap" problem. The experimental results shows that the proposed algorithm is effective and efficient on both simulate networks and real networks.
\end{abstract}

Keywords: genetic-based; link clustering; overlapping communities; community detection

\section{$1 \quad$ Introduction}

Many complex systems in nature and society can be described in terms of networks or graphs. The study of networks is crucial to understanding both the structure and the function of these complex systems. Researchers found that a common feature which is called community structure exists in many complex networks. Community structures are always expressed as clusters of nodes with dense connections within cluster and sparse connections with the other clusters. The community structure plays an important role in the complex network which can help people to understand the function of the complex network and find the potential law in the complex network. Take the World Wide Web as an example, close hyperlink web pages form a community and they often talk about related topics.

The identification of community structure has attracted much attention from various scientific fields. A lot of algorithms have been proposed for detecting communities in complex networks. The traditional community detection algorithm is to divide the complex network into several disconnected communities (or clusters, groups, 
etc.), and each node must be affiliated with one community. The representative algorithms include the modularity optimization algorithm[1-2], spectral clustering method[3-4], and so on. However, there are many overlapping networks in real world. That is to say, in the complex networks, some nodes can't belong to only one community, they can belong to multiple communities at the same time. For example, in a social network, each person can belong to more than one social group at the same time (e.g., school, family, friends, etc).

Recently, the overlapping community structure has been widely studied. Some algorithms use the clique percolation to detect the overlapping community, such as the well-known CPM[5], SCP[6] and EAGLE[7]. Some algorithms utilize the local expansion by optimizing a local benefit function, such as LFM[8], MONC[9], CIS[10] and OSLOM[11]. Some label propagation based algorithms allow multiple labels for each node to detect overlapping structure, such as COPRA[12], SLPA [13], etc. Some algorithms are Based on the link clustering, such as LINK[14], Link Maximum Likelihood[15] and Link-Comm[16]. Although the overlapping community detection has obtained significant achievements, with the network structure increasingly complex, the community detection is more difficult. how to more accurately and effectively detect the overlapping community structure is still a great challenge.

In this paper, we propose an improved genetic-based link clustering for overlapping community detection. Firstly, the algorithm changed the node graph into the link graph. Secondly, the algorithm adopted the genetic algorithm to detect the link communities. Thirdly, the algorithm transformed the link communities into the node communities. Automatically, the nodes, which are linked with edges belonged to different link communities, will be the overlapping nodes. Last, in order to improve the quality of community detection, we defined an effective method to solve the "excessive overlap" problem. The effectiveness of the proposed algorithm is demonstrated by extensive tests on both simulate networks and real networks with a known community structure. Through experimental comparison, the proposed algorithm is effective and efficient in overlapping community detection.

\section{Related work}

The genetic algorithm for overlapping community detection (GaoCD)[17] was newly proposed in 2013. In this paper, they proposed a genetic algorithm for overlapping community detection based on the link clustering. Different from those node-based overlapping community detection algorithms, the GaoCD algorithm applies a novel genetic algorithm to cluster on the edge set of network. The genetic representation and the corresponding operators effectively represent the link communities and make the number of the communities determined automatically. In the GaoCD algorithm, it mainly includes three components: objective function, genetic representation and genetic operators. 


\subsection{Objective function}

In the GaoCD algorithm, the partition density $D$ is utilized to evaluate the link density within communities. The partition density $D$ is proposed in the LINK algorithm[10], which emphasizes the community density and ignores the connection among communities. the partition density $D$ is defined as follows.

For a network with $M$ links and $N$ nodes, $P=\left\{P_{1}, P_{2}, \ldots, P_{\mathrm{c}}\right\}$ is a partition of the links into $C$ subsets. The number of links in subset $P_{\mathrm{c}}$ is $m_{\mathrm{c}}$. The number of induced nodes, all nodes that those links touch, is $n_{\mathrm{c}}$.

$$
D=\frac{2}{M} \sum_{c} m_{c} \frac{m_{c}-\left(n_{c}-1\right)}{\left(n_{c}-2\right)\left(n_{c}-1\right)}
$$

\subsection{Objective function}

In the GaoCD algorithm, a gene represents a link. An individual gene sequence in the population is represented as a gene type $\left[g_{0}, g_{1}, \ldots, g_{\mathrm{i}}, \ldots, g_{\mathrm{m}-1}\right]$. Among them, the $m$ is the number of the edges in the network, $i \in[0, m)$ is the identifier of edges in the network, and each $g_{\mathrm{i}}$ is a random adjacent edge of edge $i$.

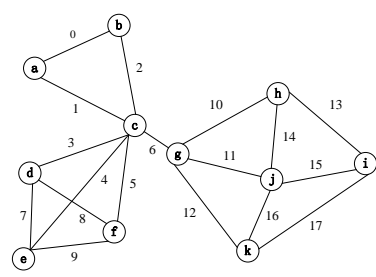

(a) The example network

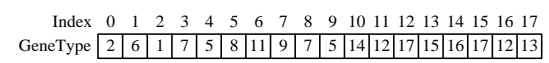

(b) The genetic representation

Fig. 1. Illustration of the genetic representation

For example, in Fig. 1.(a), $e_{0}$ has two adjacent edges $e_{1}$ and $e_{2}$. So the $e_{1}$ is the possible value of $g_{0}$. The encoding schema guarantees that every community partition can be encoded into a corresponding gene type and every gene type can be decode into an valid community partition. What's more, the encoding schema can automatically determine the number of the communities, without any prior information.

\subsection{Genetic operation}

According to the genetic representation, The GaoCD algorithm adopts the corresponding genetic operators.

In the crossover operation, They randomly select two individuals from the current population. The exchanging positions are randomly generated and then exchange the genes in these positions between these two individuals. Since the $g_{\mathrm{i}}$ is always the iden- 
tity of the adjacent edges of $e_{\mathrm{i}}$, the exchanged individuals also follow the genetic representation rule: $g_{\mathrm{i}}$ is an adjacent edge of $e_{\mathrm{i}}$.

In the mutation operation, an individual is randomly selected from the current population and the positions are randomly generated. Then they reassign the gene values on these positions with a random adjacent edge.

\section{An improved genetic-based link clustering for overlapping community detection}

The GaoCD algorithm can effectively reveal overlapping structure. However, the GaoCD algorithm is also easy to appear the "excessive overlap" problem.

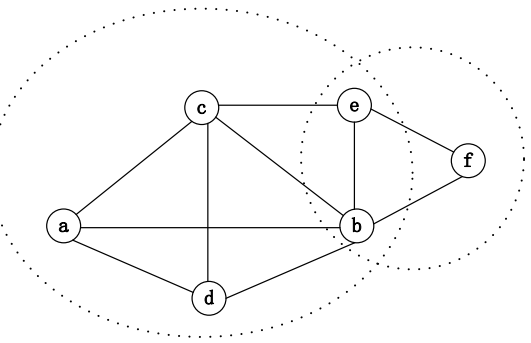

(a)

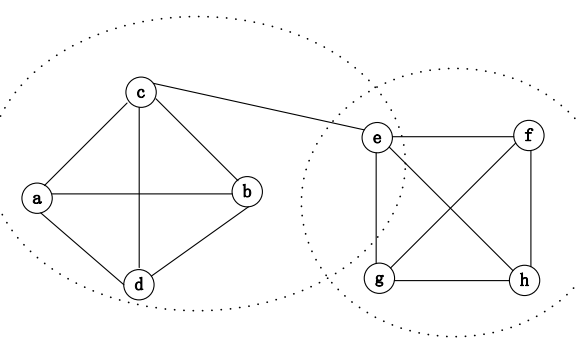

(b)

Fig. 2. Illustration of the "excessive overlap" problem

For example, the Fig. 2. is two kinds schematic diagrams of the "excessive overlap" problem. In the Fig. 2 (a), all nodes should be divided into only one community, however, they are divided into two communities, making the node e and node $\mathrm{b}$ become the overlapping nodes. In the Fig. 2 (b), the node e should only belong to the right community, however, it belongs to the both right community and left community.

In order to avoid the "excessive overlap" problem and improve the community detection performance. we proposed an improved genetic-based link clustering for overlapping community detection.

\subsection{Community similarity}

To solve the "excessive overlap" problem as shown in Fig.2(a), we define a community similarity to measure the contact ratio of communities.

Definition 1. Community Similarity

Given two communities $C 1$ and $C 2$, the community similarity is define as

$$
S\left(C_{1}, C_{2}\right)=\frac{\left|C_{1} \cap C_{2}\right|}{\min \left(\left|C_{1}\right|,\left|C_{2}\right|\right)}
$$


Given a set of communities $C S$ and a community $C$, we can define the nearduplicates of $C$ to be all communities in $C S$ that are within a contact ratio $\Delta$, where $\Delta$ is the maximum community similarity threshold. When the community similarity of the two communities is beyond threshold $\Delta$, the two communities will be merged. In the experiment, we found that it is very reasonable that this threshold $\Delta$ be set at about 0.66 . The algorithm of calculating community similarity is shown in algorithm 1 .

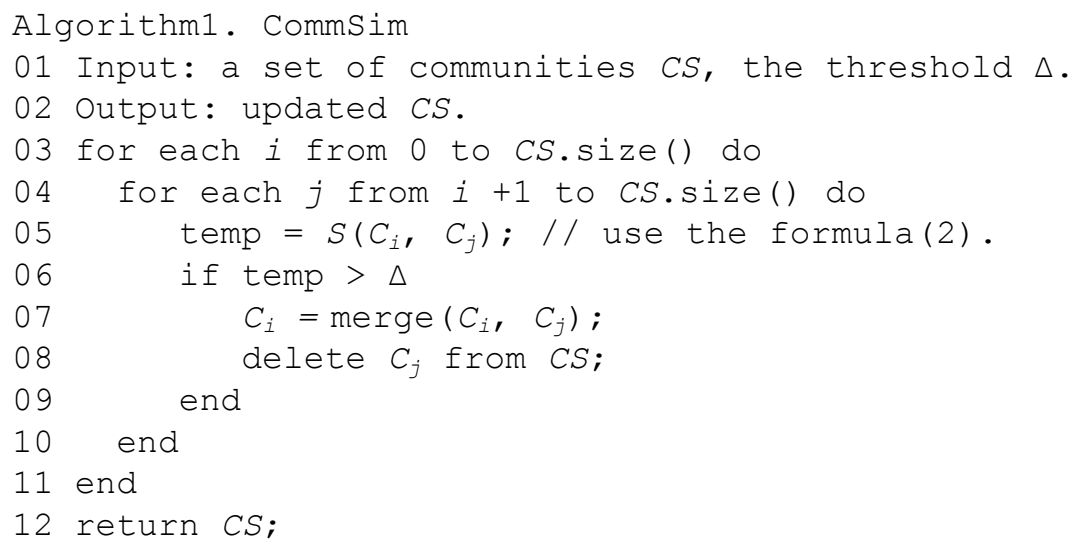

\subsection{Belonging coefficients}

To solve the "excessive overlap" problem as shown in Fig.2(b), we define the belonging coefficients to decide that the overlapping nodes belong to multiple communities or only a single community.

\section{Definition 2. Belonging Coefficients}

Given a community $C$ and an overlapping node $v$, belonging coefficients is defined as fallow:

$$
B C(v, C)=\frac{|E(v)||E(v)|}{|E(C)||K(v)|}
$$

Among them, the node $v$ denotes an overlapping node which belongs to community $C$. $E(v)$ denotes the edges which connect node $v$ to the community $C$. $E(C)$ denotes the edges in the community $C . K(v)$ denotes the degree of node $v$.

For the nodes with multiple memberships, we use the belonging coefficients to determine whether nodes are excessive overlap nodes. In order to facilitate comparison, we introduce a threshold $\tau$, where $\tau$ is the maximum difference between two belonging coefficients in different communities. In the experiment, we found that it is very reasonable that this threshold $\tau$ be set at about 0.25 . The algorithm about the belonging coefficients is shown in algorithm 2. 


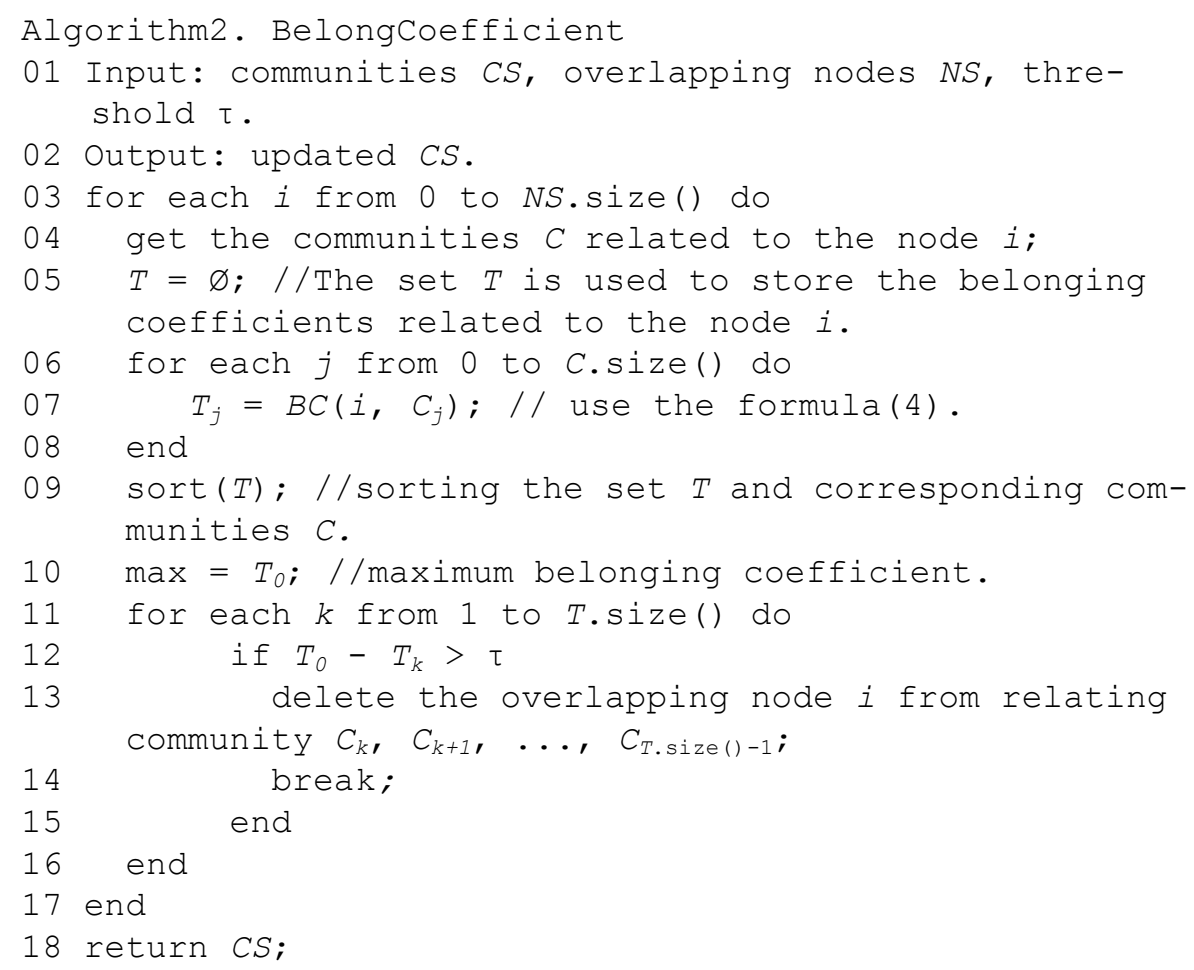

\section{$4 \quad$ Experiments}

In this section, the IGLC algorithm is tested on the simulated data sets and real data sets, respectively. Experimental environment: Processor Inter (R) Core (TM) i5 3.1GHz PC, memory 4G, the operating system is Windows 7, programming environment Matlab R2009a.

\subsection{Experimental data sets}

The simulated data sets.

Currently, the LFR benchmark network[18-19] is the most commonly used data set in community detection. We generate two LFR benchmark networks, whose detail information are shown in Table 1. Some important parameters of the benchmark networks are as follow:

$N$ : the number of nodes; $k$ : the average degree; maxk: the maximum degree; minc: the minimum for the community sizes; $\operatorname{maxc}$ : the maximum for the community sizes; on: the number of overlapping nodes; $m u$ : mixing degree; om: the number of communities that each node can belong to; 
Table 1. The LFR benchmark networks

\begin{tabular}{ccccccccc}
\hline Num & $N$ & $k$ & $\operatorname{maxk}$ & $\operatorname{minc}$ & $\operatorname{maxc}$ & on & mu & om \\
\hline S1 & 1000 & 20 & 50 & 10 & 50 & 100 & 0.1 & $2 \sim 8$ \\
S2 & 1000 & 20 & 50 & 10 & 50 & 100 & 0.3 & $2 \sim 8$ \\
\hline
\end{tabular}

The real data sets.

We make experiments on five well known social networks, whose real community structure have been given. Their specific information is shown in Table 2.

Table 2. The real network

\begin{tabular}{cccc}
\hline Name & Nodes & Edges & Source \\
\hline Karate & 34 & 78 & {$[20]$} \\
Dolphins & 62 & 159 & {$[20]$} \\
Political Books & 105 & 441 & {$[20]$} \\
Football & 115 & 613 & {$[20]$} \\
Netscience & 379 & 914 & {$[20]$} \\
\hline
\end{tabular}

\subsection{Evaluation criteria}

In the experiments, we use the evaluation criteria normalized mutual information $(N M I)[12]$ and extended modularity $(E Q)[7]$ to evaluate the communities.

\section{Normalized mutual information (NMI).}

The NMI is used to measure similarity between the results of algorithm with true class values.

Assuming that the true class values of the data sets are $C=\left\{C_{1}, C_{2}, \ldots, C_{\mathrm{k}}\right\}$, and the class labels obtained by the algorithm are $U=\left\{U_{1}, U_{2}, \ldots, U_{1}\right\}$, where $k$ and $l$ denote the number of clusters in $C$ and $U$. The number of nodes in the $C i(1 \leq i \leq k)$ and $U j(1 \leq j \leq l)$ are $n_{\mathrm{i}}$ and $n_{\mathrm{j}}$ respectively. The length of intersection of $C_{\mathrm{i}}$ and $U_{\mathrm{j}}$ is $n_{\mathrm{ij}}$, so $N M I$ is defined as Eq.(4-5).

$$
\begin{gathered}
N M I=\frac{2 \times I(C, U)}{H(C)+H(U)} \\
N M I=\frac{-2 \sum_{i=1}^{k} \sum_{j=1}^{l} \frac{n_{i j}}{n} \log \frac{n_{i j}}{n_{i} \times n_{j}}}{\sum_{i=1}^{k} n_{i} \log \frac{n_{i}}{n}+\sum_{j=1}^{l} n_{j} \log \frac{n_{j}}{n}}
\end{gathered}
$$

\section{Extended modularity(EQ) .}


The $E Q$ is a variant of the commonly used modularity $(Q)$ metric[1], which is defined for overlapping communities by Shen. This extended modularity is defined as follow:

$$
E Q=\frac{1}{2 m} \sum_{C} \sum_{i, j \in C_{k}} \frac{1}{O_{i} O_{j}}\left[A_{i j}-\frac{k_{i} k_{j}}{2 m}\right]
$$

\subsection{Experimental results}

In the experiments, we use two algorithms to compare with the proposed IGLC algorithm. The two algorithms are COPR[12] and LINK[14], respectively. The parameters of IGLC are set as follows: size $=100$, gens $=100, p c=0.6, p m=0.4, \Delta=0.66, \tau=$ 0.25 . The parameter of COPRA is set as follows: $v=4$. The LINK algorithm don't need parameters.

\section{The results on the simulated data sets.}

The results on the two simulated data sets are shown in the Fig. 3. The abscissa is the om whose value ranges from 2 to 8 , and the ordinate is the NMI value. The NMI value of per om is the average of 10 times.

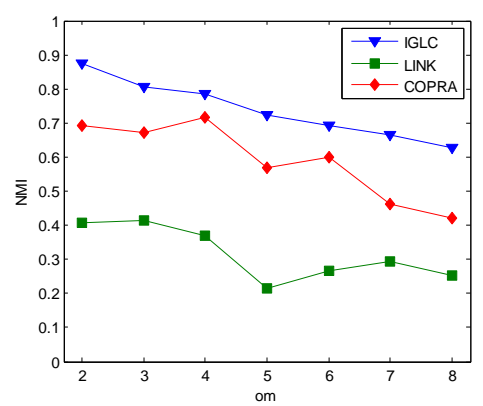

(a) $\mathrm{S} 1(m u=0.1)$

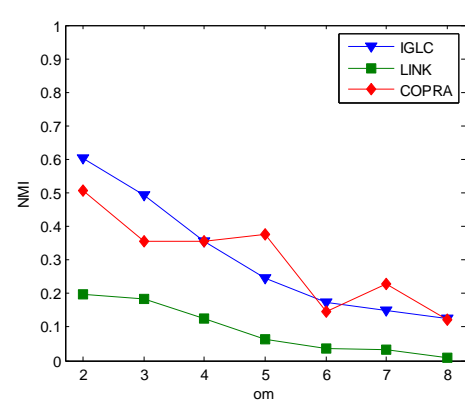

(b) $\mathrm{S} 2(m u=0.3)$

Fig. 3. The results on the simulated data sets

(1) Compared with the LINK algorithm.

In the two LFR benchmark networks, the results of IGLC are all better than the results of LINK. Because the LINK algorithm exists "excessive overlap" problem, which seriously reduces the quality of community detection. However, the proposed IGLC algorithm solves the "excessive overlap" problem very well. Therefore, the proposed IGLC algorithm effectively improves the quality of community detection.

(2) Compared with the COPRA algorithm.

In the low mixing degree LFR benchmark network $(m u=0.1)$, the $N M I$ values of IGLC are all better than the NMI values of COPRA. In the high mixed degree LFR benchmark network $(m u=0.3)$, the $N M I$ values of IGLC are most better than the NMI 
values of COPRA, Only in a few cases, the COPRA has higher NMI value(e.g. om = $(5,7)$ in the $\mathrm{S} 1$. In addition, with the increase of $\mathrm{om}$, the community detection is becoming more and more difficult. The NMI value of COPRA present fluctuations, which demonstrates that the COPRA algorithm has poor robustness. However, the $N M I$ value of proposed IGLC algorithm present the steady downward trend, which demonstrates that our algorithm has good robustness.

In conclusion, the proposed IGLC algorithm can obtain better quality of overlapping community detection compared with the LINK algorithm and COPRA algorithms in most cases.

\section{The results on the real data sets.}

Table 3 shows community detection results of three algorithms on real data sets, whose detailed information is shown in Table 2 . The bold in each row is the optimal community detection result. The evaluation criterion in Table 3 is the extended modularity $E Q$.

Table 3. The results on the real network

\begin{tabular}{cccc}
\hline Name & LINK & COPRA & IGLC \\
\hline Karate & 0.146 & 0.423 & $\mathbf{0 . 5 1 4}$ \\
Dolphins & 0.351 & 0.683 & $\mathbf{0 . 7 2 9}$ \\
Political Books & 0.254 & $\mathbf{0 . 8 1 3}$ & 0.804 \\
Football & 0.557 & 0.685 & $\mathbf{0 . 6 8 9}$ \\
Netscience & 0.457 & 0.812 & $\mathbf{0 . 8 9 3}$ \\
\hline
\end{tabular}

In the Table 3, compared with the LINK and COPRA algorithm, the proposed IGLC algorithm can obtain better clustering results on most networks. Although the proposed IGLC algorithm don't have the best $E Q$ values on the Political Books network, The $E Q$ values are also the second best values.

In conclusion, the proposed IGLC algorithm can achieve acceptable results on real data sets, so the IGLC algorithm is reasonable and effective in overlapping community detection.

\section{Conclusion}

In this paper, we propose an improved genetic-based link clustering for overlapping community detection(IGLC). The IGLC algorithm mainly includes two parts. One part is adopting the GaoCD algorithm to detect the link communities. The other is transforming the link communities into the node communities and adopting the community similarity and the belonging coefficients to solve the "excessive overlap" problem. Through experimental comparison, the proposed algorithm is effective and efficient on both simulate networks and real networks. 


\section{$6 \quad$ References}

1. Newman M E J, Girvan M. Finding and evaluating community structure in networks[J]. Physical review E, 2004, 69(2): 026113.

2. Lee J, Gross S P, Lee J. Modularity optimization by conformational space annealing[J]. Physical Review E, 2012, 85(5): 056702.

3. Shen H W, Cheng X Q. Spectral methods for the detection of network community structure: a comparative analysis[J]. Journal of Statistical Mechanics: Theory and Experiment, 2010, 2010(10): P10020.

4. Jiang J Q, Dress A W M, Yang G. A spectral clustering-based framework for detecting community structures in complex networks[J]. Applied Mathematics Letters, 2009, 22(9): 1479-1482.

5. Palla G, Derényi I, Farkas I, et al. Uncovering the overlapping community structure of complex networks in nature and society[J]. Nature, 2005, 435(7043): 814-818.

6. Kumpula J M, Kivelä M, Kaski K, et al. Sequential algorithm for fast clique percolation[J]. Physical Review E, 2008, 78(2): 026109.

7. Shen H, Cheng X, Cai K, et al. Detect overlapping and hierarchical community structure in networks[J]. Physica A: Statistical Mechanics and its Applications, 2009, 388(8): 17061712.

8. Lancichinetti A, Fortunato S, Kertész J. Detecting the overlapping and hierarchical community structure in complex networks[J]. New Journal of Physics, 2009, 11(3): 033015.

9. Havemann F, Heinz M, Struck A, et al. Identification of overlapping communities and their hierarchy by locally calculating community-changing resolution levels[J]. Journal of Statistical Mechanics: Theory and Experiment, 2011, 2011(01): P01023.

10. Kelley S. The existence and discovery of overlapping communities in large-scale networks[M]. RENSSELAER POLYTECHNIC INSTITUTE, 2009.

11. Lancichinetti A, Radicchi F, Ramasco J J, et al. Finding statistically significant communities in networks[J]. PloS one, 2011, 6(4): e18961.

12. Gregory S. Finding overlapping communities in networks by label propagation[J]. New Journal of Physics, 2010, 12(10): 103018.

13. Xie J, Szymanski B K, Liu X. Slpa: Uncovering overlapping communities in social networks via a speaker-listener interaction dynamic process[C]//Data Mining Workshops (ICDMW), 2011 IEEE 11th International Conference on. IEEE, 2011: 344-349.

14. Ahn Y Y, Bagrow J P, Lehmann S. Link communities reveal multiscale complexity in networks[J]. Nature, 2010, 466(7307): 761-764.

15. Ball B, Karrer B, Newman M E J. Efficient and principled method for detecting communities in networks[J]. Physical Review E, 2011, 84(3): 036103.

16. Kim Y, Jeong H. Map equation for link communities[J]. Physical Review E, 2011, 84(2): 026110.

17. Shi C, Cai Y, Fu D, et al. A link clustering based overlapping community detection algorithm[J]. Data \& Knowledge Engineering, 2013, 87: 394-404.

18. Lancichinetti A, Fortunato S, Radicchi F. Benchmark graphs for testing community detection algorithms[J]. Physical Review E, 2008, 78(4): 046110.

19. Lancichinetti A, Fortunato S. Benchmarks for testing community detection algorithms on directed and weighted graphs with overlapping communities[J]. Physical Review E, 2009, 80(1): 016118.

20. Newman. Network Data [EB/OL]. http://www-personal.umich.edu/- mejn/netdata/. 2013-4-19. 